\title{
Comparison of clinical and self reported diagnosis for rheumatology outpatients
}

\author{
Iris Rasooly, Ann C Papageorgiou, Elizabeth M Badley
}

\begin{abstract}
Objective-To examine the sensitivity of patient self reported diagnoses compared with physician diagnoses in a rheumatology outpatient population.

Methods-A mailed survey to 472 rheumatology outpatients ( $81 \%$ response rate) asked about joint symptoms, disabilities, and underlying rheumatic conditions. The self-reported diagnoses were linked with physician diagnoses in the rheumatology clinic computer based diagnostic registry. Result-Overall there was an $87 \%$ sensitivity for self reported compared with physician diagnoses when the matching criteria included compatible yet different diagnoses such as rheumatoid arthritis (RA) and osteoarthritis (OA). The sensitivity for exact match was $65 \%$, and it varied with the underlying clinical diagnosis, and was greatest for RA $(90 \%)$ and ankylosing spondylitis (AS) $(100 \%)$, and intermediate for OA $(52 \%)$ and psoriatic arthritis $(50 \%)$. The sensitivity of self report was primarily related to the type of diagnosis (RA or AS $v$ other rheumatic conditions; odds ratio $=16.3,95 \%$ confidence interval (CI) $9 \cdot 0$ to $29 \cdot 5$ ), and also to difficulty in activities of daily living (odds ratio $=2 \cdot 3,95 \%$ CI $1 \cdot 1$ to $4 \cdot 6$ ) but not age, gender, duration of disease, or clinic attendance, as shown by multivariate analysis.
\end{abstract}

Conclusions-This study in a rheumatology outpatient population indicated that most patients report a diagnosis which is compatible with the clinical diagnosis. These findings give an upper limit to the sensitivity of self reported diagnoses, though further research is needed to assess the extent to which our results may be generalised to other settings.

(Ann Rheum Dis 1995; 54: 850-852)

Our knowledge about the occurrence of rheumatic disorders in the population comes mainly from health surveys which use respondents' self report to identify health conditions, for example in the United Kingdom, ${ }^{1}$ Canada, ${ }^{2}$ and the USA. ${ }^{3}$ The validity or accuracy of patient reported conditions has been examined in only a few studies; ${ }^{4-10}$ these found that, in general, major chronic health conditions (for example cancer and heart disease) were reported accurately by patients. Little is known, however, about the accuracy of patient reported rheumatic conditions. ${ }^{10}$

The purpose of the present study was to examine the accuracy of patient reported rheumatic diagnosis compared with the diagnosis recorded by the physician in the context of a rheumatology clinic having a well kept diagnostic registry with which to compare patients' self reported diagnoses.

\section{Patients and methods}

All 472 patients who attended the rheumatology outpatient clinic at Manchester Royal Infirmary, a university hospital, during December 1985 and who had a diagnosis recorded in the clinic diagnostic registry were sent a four page questionnaire in May 1986. A reminder was sent to non-responders after three weeks. The questionnaire was designed to screen for disability and joint symptoms, ${ }^{1}{ }^{11}$ and had two separate sections in which up to three self reported diagnoses could be recorded. The first section asked responders who indicated physical disabilities to write in their main illness or disability. The second section, focusing on problems with joints, provided a check list of 17 common kinds of arthritis and rheumatism, though patients could enter other conditions or 'don't know' as appropriate.

The data obtained through the survey were linked to the computerised diagnostic registry of the rheumatology clinic. This included information on each patient's year of birth, gender, clinical diagnosis, year of disease onset, and date of first clinic attendance. All diagnoses were coded to four digit categories of the International Classification of Diseases (9th revision) (ICD-9). ${ }^{12}$ Rheumatic diagnoses were defined as those included in chapter XIII (Musculoskeletal System and Connective Tissue) of the ICD-9, plus temporal arteritis and Raynaud's syndrome. In calculating the number of diagnoses reported, duplicate codes and non-rheumatic diagnoses were excluded.

Patient reported diagnoses were compared with the recorded clinical diagnosis using the criteria for matching outlined below. When more than one rheumatic diagnosis was reported or recorded, the primary match was between those closest.

(1) Exact match: Identical disease entities at the four digit level.

(2) Crude match: Compatible self report and clinical diagnoses within the four major subsections in ICD chapter XIII (a) arthropathies, (b) dorsopathies, (c) rheumatism excluding the back (disorders of muscles 
Table 1 Characteristics of the study population $(n=373) \dagger$

\begin{tabular}{lc}
\hline Characteristic & $\begin{array}{l}\text { Frequency (\%) } \\
\text { or Mean } \\
\text { (interquartile } \\
\text { range) }\end{array}$ \\
\hline Clinical data & \\
Age (yr) & $55(46-65)$ \\
Female gender & $250(67 \%)$ \\
Duration of illness (yr) & $9 \cdot 7(2-13)$ \\
Period attending clinic (yr) & $6 \cdot 4(1-8)$ \\
Clinical diagnosis $\ddagger$ & $315(84 \%)$ \\
Arthropathies & $36(10 \%)$ \\
Soft tissue rheumatism & $22(6 \%)$ \\
Dorsopathies & $81(21 \%)$ \\
Other non-rheumatic diagnosis & $362(97 \%)$ \\
Self report questionnaire data & $308(83 \%)$ \\
Joint symptoms & $111(30 \%)$ \\
Has difficulty $\$$ & $336(90 \%)$ \\
Has dependence $\$$ & $28(8 \%)$ \\
Self reported rheumatic diagnosis $\uparrow$ & Self reported non-rheumatic diagnosis $\uparrow$ \\
\hline
\end{tabular}

†Excludes eight respondents whose recorded clinical diagnoses were respectively: myxoedema, atherosclerosis, post myocardial infarction syndrome, angina, dystonia, Wegner's granulomatosis, hyperkinetic syndrome, and not stated.

$\ddagger$ Percentages add to $>100 \%$ as more than one diagnosis could be recorded.

IIn at least one activity of daily living.

ףIn association with disability or joint symptom.

tendons and other soft tissues), or (d) osteopathies, chondropathies and musculoskeletal deformities.

(3) No match: Diagnoses from different subsections of chapter XIII, for example arthritis and osteoporosis.

(4) Patient reported diagnosis missing.

The percent of patients for whom the self reported diagnosis matched the clinical diagnosis represents the sensitivity of self reported diagnosis with respect to the clinical diagnosis. Sensitivity was calculated for both exact match and any match (exact and crude).

Statistical analysis used the SPSS/PC $+4 \cdot 0$ statistical package. ${ }^{13}$ For bivariate analyses, $\chi^{2}$ or $t$ test were used as appropriate, and logistic regression was used for the multivariate analysis.

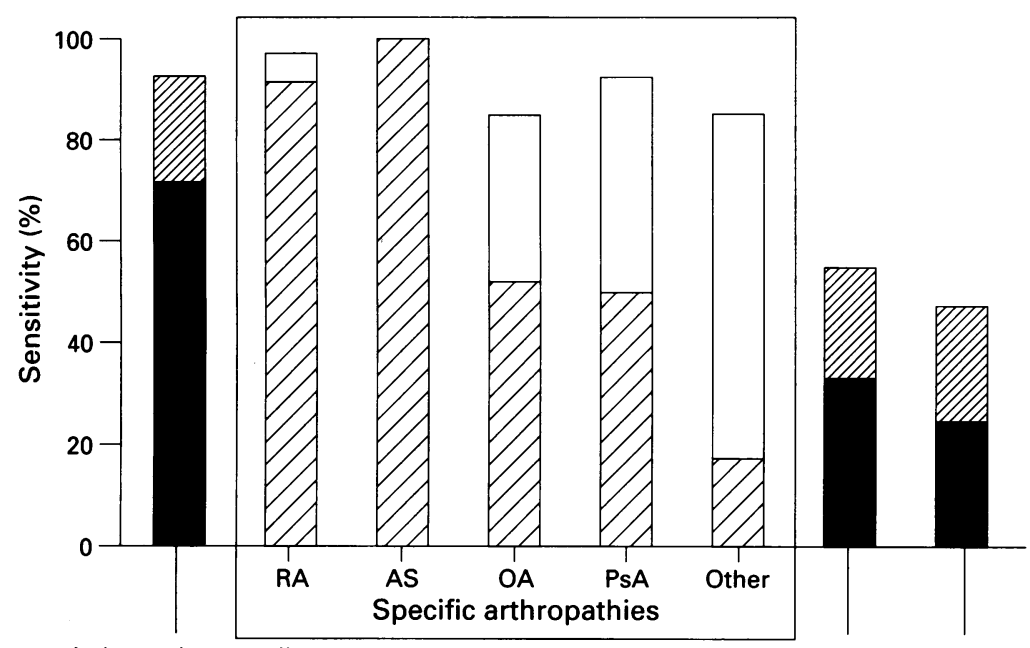

Arthropathy overall

Dorsopathy Rheumatism

Figure 1 Sensitivity of patient self reported rheumatic diagnoses compared with physician diagnoses. Match: $\square$ or $\square=$ crude; $\square$ or $\square=$ exact. 'Arthropathy overall' comprises: rheumatoid arthritis 191; ankylosing spondylitis 18; osteoarthritis 13; psoriatic arthritis 14; other arthropathy 59. 'Other arthritis' comprises: polyarthritis 22; specific arthropathies 17 (systemic lupus erythematosus 1; scleroderma 3; Sjögren's syndrome 2; Reiter's syndrome 2; gout 6; gastrointestinal arthropathy 1; palindromic rheumatism 2); non-specific

arthropathies 20 (traumatic 1; unspecified 9; joint pain 10). 'Rheumatism' comprises rheumatism, osteopathies and other rheumatic disorders 36 (carpal tunnel syndrome 1 , Raynaud's syndrome 3; temporal arteritis 2; polymyalgia rheumatica 7; shoulder enthesopathy 4; elbow enthesopathy 1; ankle enthesopathy 1; synovitis 5; hypermobility 1; unspecified rheumatism 2; myalgia 1; faciitis 1; limb pain 1; osteoporosis 1; aseptic necrosis 1 ; flat foot 2; osteodystrophy 1; sprain/strain 1).

\section{Results}

Of a total of 472 questionnaires, $381(81 \%)$ were completed after one reminder and a further 10 were returned uncompleted (one person refused to participate, three had died, and six had moved away). Responders were slightly older (median age 55) than nonresponders (median age 49) and were more likely to have rheumatoid arthritis $(p>0.01)$. Table 1 summarises the characteristics of the 373 patients included in the analysis, all of whom had a recorded clinical diagnosis of a rheumatic disorder.

The clinical diagnoses recorded in the study population were predominantly arthropathies; the most frequently recorded were rheumatoid arthritis (RA) (51\% of the study population), osteoarthritis (OA) (9\%), ankylosing spondylitis (AS) $(5 \%)$, and psoriatic arthritis (PsA) $(4 \%)$. More than one clinical rheumatic diagnosis was recorded for 46 patients $(12 \%)$; these were mainly combinations of different types of arthropathies.

For rheumatic conditions overall, the sensitivity of self reported diagnosis compared with the clinical diagnosis was $65 \%$ for an exact match, and this increased to $87 \%$ when a crude match was included. Thirty seven patients $(10 \%)$ either did not report a rheumatic diagnosis (16) or indicated that they did not know (21). Only 3\% of patients indicated a diagnosis which did not at all match that of the physician.

The sensitivity of self reported diagnosis varied for the different rheumatic conditions, as presented in the figure. It was greatest within the arthropathy group, where a sensitivity of $93 \%$ was found using any match and $72 \%$ using exact match. Among the specific arthropathies there was also variation in sensitivity, as indicated in the inset box in the figure. Sensitivity was very high for RA and AS, and intermediate for OA and PsA. Other arthropathies, as detailed in the footnote to the figure, had a lesser pooled sensitivity for exact match $(17 \%)$, but sensitivity was $85 \%$ when crude matches were included. For dorsopathies and for soft tissue rheumatism, the sensitivities were $57 \%$ and $47 \%$, respectively, using any match. For both these groups of condition, no self report diagnosis was given in approximately $30 \%$ of cases.

To explore the factors which might influence sensitivity, we compared patients in whom there was an exact match between the self reported and clinical diagnoses with those having either a crude match only, no match, or missing patient response (table 2 ). Those with exact match had a longer disease duration, longer duration of clinic attendance, a greater severity of joint symptoms as indicated by the number of joints involved, and more difficulty or dependence in activities of daily living. However, there were no significant differences in age or gender between the two groups.

The independent contribution of the different variables associated with the precision of match was investigated using multivariate logistic regression analysis. The type of clinical diagnosis was the most powerful predictor of 
Table 2 Variables associated with precision of match: univariate analysis

\begin{tabular}{llll}
\hline Characteristic & \multicolumn{2}{l}{ Mean $(S D)$ or \% in patients with } & \multirow{2}{*}{$p$} \\
\cline { 2 - 3 } & Exact match & Crude or no match & \\
\hline Type of diagnosis (\% RA/AS) $\dagger$ & $73 \%$ & $16 \%$ & $<0 \cdot 00001$ \\
Duration of illness (yr) & $10 \cdot 7(8 \cdot 3)$ & $7 \cdot 4(5 \cdot 6)$ & $<0 \cdot 001$ \\
Period attending clinic (yr) & $7 \cdot 4(7 \cdot 7)$ & $4 \cdot 2(5 \cdot 3)$ & $<0 \cdot 001$ \\
Number of joints involved & $5 \cdot 8(2 \cdot 6)$ & $4 \cdot 4(2 \cdot 6)$ & $<0 \cdot 001$ \\
Has difficultył & $89 \%$ & $69 \%$ & $<0 \cdot 0001$ \\
Has dependence & $33 \%$ & $23 \%$ & $0 \cdot 04$ \\
Age (yr) & $56(13)$ & $53(15)$ & $\mathrm{NS}$ \\
Female gender & $69 \%$ & $63 \%$ & $\mathrm{NS}$ \\
\hline
\end{tabular}

†Rheumatoid arthritis (RA) or ankylosing spondylitis (AS).

In at least one activity of daily living.

the precision of match (odds ratio $=16 \cdot 3,95 \%$ confidence interval (CI) $9 \cdot 0$ to $29 \cdot 5$ ). Of the other five variables significantly associated with precision of match in the bivariate analysis, only having difficulties in daily living activities remained significantly associated with precision of match in the multivariate analysis (odds ratio $=2 \cdot 3,95 \%$ CI $1 \cdot 1$ to $4 \cdot 6$ ).

\section{Discussion}

In this study, of a rheumatology outpatient population, we found self reported rheumatic diagnosis had an overall sensitivity, across broad diagnostic groups, of $87 \%$, with considerable variation among different rheumatic diseases.

Sensitivity represents the proportion of clinical diagnoses correctly self reported (true positives). It was not appropriate to calculate specificity, the proportion of 'true negatives', as a clinical population is essentially a selected population-all non-rheumatic cases are missing. It should be noted that very few patients $(3 \%)$ indicated an incorrect diagnosis (termed 'no match'); most incongruities consisted of reporting a related diagnosis ('crude match'-partially correct) or not indicating one at all.

Our study setting may provide an upper estimate of self report sensitivity. Certain individual conditions for which self report sensitivity was high (for example rheumatoid arthritis) were overrepresented in our outpatient population, thus increasing the overall sensitivity. The greater disease severity presumed to exist in our outpatient population, and the better medical information we assumed to be provided to them, could also positively effect the completeness and accuracy of reporting by these patients. ${ }^{9}$ Further, in patients for whom more than one condition was reported we took the closest match, which could give an unduly optimistic picture. Nevertheless, our findings are similar to those observed in a general practice setting for osteoarthritis and rheumatoid arthritis. ${ }^{10}$

The availability of a diagnostic registry facilitated our estimation of sensitivity as this permitted us to corroborate patients' self reported diagnosis. In the absence of such a registry, missing clinical information ${ }^{1014}$ to which the patient may have been referring could lead to underestimation of self report accuracy. ${ }^{6}{ }^{15}$ The wording of questions may also have contributed to patients' recall, especially the list of 17 specific rheumatic disorders which followed the open ended question on diagnosis.

Last, when comparing self reported diagnosis with the clinical diagnosis, it is important to be aware that the latter is not a true 'gold standard'. There is a lack of uniformity among physicians in determining a clinical diagnosis even for the conditions for which diagnostic criteria have been formulated. As clinical practice does not usually include the standardised use of diagnostic criteria, applying such criteria post factum to clinical information might lead to underestimation of the sensitivity of self report, as patients would still report the condition they were told they had, regardless of its redefinition within the study. ${ }^{5}$

This study found good sensitivity of self report, both overall and within broad subgroups of rheumatic conditions. Patients rarely reported a diagnosis that was incompatible with their clinical one. These findings give an upper estimate of sensitivity for self report. Further research is required to determine the extent to which our findings may be generalised to other settings.

We wish to thank Dr P J L Holt for allowing us access to patients and for his valuable help in carrying out this study. Thanks are also due to Professor P H N Wood for his helpful comments and support, and to the staff of the ARC Epidemiology Unit for assistance with data handling.

1 Badley E M, Tennant A. Impact of disablement due to rheumatic disorders in a British population: estimates of severity and prevalence from the Calderdale Rheumatic Disablement Survey. Ann Rheum Dis 1993; 52: 6-13.

2 Reynolds D L, Chambers L W, Badley E M, et al. Physical disability among Canadians reporting musculoskeletal diseases. $\mathcal{f}$ Rheumatol 1992; 19: 1020-30.

3 Kelsey J L. Epidemiology of musculoskeletal disorders. New York: Oxford University Press. 1982

4 Paganini-Hill A, Ross R K. Reliability of recall of drug usage and other health-related information. $A m \mathcal{F}$ Epidemiol and other health-rel.

5 Tretli S, Lund-Larsen P G, Foss O P. Reliability of questionnaire information on cardiovascular disease and diabetes: cardiovascular diseases study in Finnmark county. F Epidemiol Commun Health 1982; 36: 269-73.

6 Meltzer J W, Hochstim J R. Reliability and validity of survey data on physical health. Public Health Reports 1970; 85: 1075-86.

7 Colditz G A, Martin P, Stampfer M J, et al. Validation of questionnaire information on risk factors and disease outcomes in a prospective cohort study of women. $A m \mathcal{F}$ Epidemiol 1986; 123: 894-900.

8 Spitz M R, Fueger J J, Newell G R. The development of a comprehensive institution-based patient risk evaluation program: II. Validity and reliability of questionnaire data. program: II. Validity and reliability

9 Jabine J B. Reporting chronic conditions in the National Health Interview Survey. Data Evaluation and Methods Research.
Series 2. No. 105. Vital \& Health Statistics. DHHS Series 2. No. 105. Vital \& Health Statistics. DHHS
publication No. (PHS) 87-1379. Washington: US publication No. (PHS) 87-1379. Washington:

10 Warren M D. Interview surveys of handicapped people: the accuracy of statements about the underlying medical condition. Rheumatol Rehabil 1976; 15: 295-302.

11 Badley E M, Tennant A. Changing profile of joint disorders with age: findings from a postal survey of the population of Calderdale, West Yorkshire, United Kingdom. Ann Rheum Dis 1992; 51: 366-71.

12 International Classification of Diseases, 1975 Revision. Geneva: World Health Organization, 1977.

13 SPSS/PC+ 4.0 Manual for the IBM PC/XT/AT and PS/2. Chicago, Illinois, USA: SPSS Inc, 1990.

14 O'Toole B I, Battistutta D, Long A, Crouch K. A comparison of costs and data quality of three survey methods: mail, telephone and personal home interview. Am ₹ Epidemiol 1986; 124: 317-28.

15 Ford A B, Folmar S J, Salmon R B, Medalie J H Roy A W, Galazka S S. Health and function in the old and very old. $₹$ Am Geriat Soc 1988; 36: 187-97. 